\title{
FIBROBLAST GROWTH FACTOR (FGF): A REVIEW
}

\author{
Dr. Ram Rattan Gupta ${ }^{1}$, Dr. Manu Gupta ${ }^{2}$, Dr. Avnika Garg ${ }^{3}$ \\ ${ }^{1}$ Reader, Department of Periodontology, Bhojia Dental College and Hospital, Distt. Solan, Himachal Pradesh (India) \\ ${ }^{2}$ Private Practitioner, Punjab (India) \\ ${ }^{3}$ PG Student, Department of Periodontology, Bhojia Dental College and Hospital, Distt. Solan, Himachal Pradesh (India) \\ Corresponding Author: \\ ${ }^{1}$ Mobile: 919872810521 Email:drgupta116@gmail.com
}

\begin{abstract}
Received :

$23^{\text {rd }}$ April, 2013

Accepted:

$2^{\text {nd }}$ July, 2013

Available online:

$25^{\text {th }}$ August, 2013

In order for periodontal regeneration to occur, progenitor cells must migrate to the denuded root surface, attach to it, proliferate and mature into an organized and functional fibrous attachment apparatus. Significant advances have been made during the last decade in understanding the factors controlling the migration, attachment and proliferation of cells. A group of naturally occurring molecules known as polypeptide growth factors in conjunction with certain matrix proteins, are key regulators of these biological events. Of these, the fibroblast growth factors (FGFs) appear to have an important role in periodontal wound healing. The purpose of this review is to summarize current information on these growth factors with emphasis on their potential implications in periodontal wound healing and regeneration.
\end{abstract}

Keywords: Regeneration, Wound Healing, Fibroblast growth factor

\section{INTRODUCTION}

Fibroblast growth factors (FGFs) are members of the heparin binding growth factor family and are polypeptides that are potent mitogens and chemoattractants for endothelial cells as well as for a variety of mesenchymal cells, including fibroblasts, osteoblasts, chondrocytes, smooth muscle cells, skeletal myoblasts and for cells of neuro-ectodermal origin. FGF have not only growth-promoting effects on most fibroblastic cell types, but it also stimulates angiogenesis, neovascularization, wound healing and cell migration. ${ }^{1,2,3}$

\section{FORM AND RECEPTOR (Figure 1)}

FGFs are a family of 9 heparin-binding polypeptides, aFGF (FGF-1), bFGF (FGF-2), int-2 (FGF-3), Kaposi sarcoma FGF (K-FGF; also known as the product of hst-1 oncogene: FGF-4), FGF-5, FGF-6, keratinocyte growth factor (KGF: FGF-7), androgen-induced growth factor (FGF-8) and FGF-9. ${ }^{4}$ They all have similar activities; mitogenicity for cells of mesodermal and neuro-ectodermal origin and potent angiogenic activity in vivo. ${ }^{3}$ The two most studied members of this family are acid FGF (a-FGF or FGF1) and basic FGF (b-FGF or FGF-2). ${ }^{5}$ Both FGF-1 and FGF-2 are single chain proteins that are proteolytically derived from different precursor molecules to generate biologically active proteins of about 15,000 molecular weight. ${ }^{3}$ FGF-1 has an isoelectric point range of 5.6-6.0 and a molecular weight of approximately 15,000 Da; FGF-2 has an isoelectric point of approximately 9.6 and a molecular weight range of 16,000-18,000Da. FGF-1 and FGF-2 have a similar spectrum of biological activities and exhibit 55\% homology in their amino acid sequence $^{1}$ and are heparin binding proteins. ${ }^{5}$ FGF-2 is considered to be more potent than FGF-1 (30-100 fold) in vitro ${ }^{1}$ and may act via the stimulation of other growth factors because it has been found to stimulate Transforming growth factor- $\beta .^{5}$ However, as far as potency is concerned, in the presence of heparin, FGF-1 and FGF-2 exhibit similar mitogenic activity. FGF-1 is related to or similar to endothelial cell growth factor, eye-derived growth factor II, heparin binding growth factor $\alpha$, brain-derived 
growth factor, retinal-derived growth factor and astroglial growth factor. FGF-2 has similar properties and is related to eye-derived growth factor I, heparin binding growth factor $\beta$, chondrosarcoma-derived growth factor, hepatoma-derived growth factor and astroglial factor- $2{ }^{1}$

FGFs are recognized by a family of cell-surface receptors that have intrinsic tyrosine kinase activity. ${ }^{6}$ Signal transducing FGF receptors are encoded by a family of related genes of which 4 members have been encoded so far i.e. FGFR-1(flg), FGFR-2(bek), FGFR-3 AND FGFR-4. Each of these receptors consists of an extracellular ligand-binding region with 2 or 3 immunoglobulin-like loops. There is a high degree of redundancy among FGFRs in terms of their binding to ligands and each bind both FGF-1 and FGF-2 with high affinity. Gingival epithelial (GE) cells express each mRNA of all 4 subtypes of FGFRs, however, the number of FGFRs on a GE cell may actually be very few. In contrast, Takayama etal in 2002 confirmed that human PDL cells express FGFR-1 and FGFR-2 mRNA, but not that of FGFR-3 or FGFR-4. It is of interest that the affinity of FGF-2 to GE cells is different from that to PDL cells. FGF-2 is anchored in the intercellular spaces of gingival epithelium via heparin sulfate and may regulate the growth and cytodifferentiation of GE cells via celltype specific receptors. ${ }^{7}$

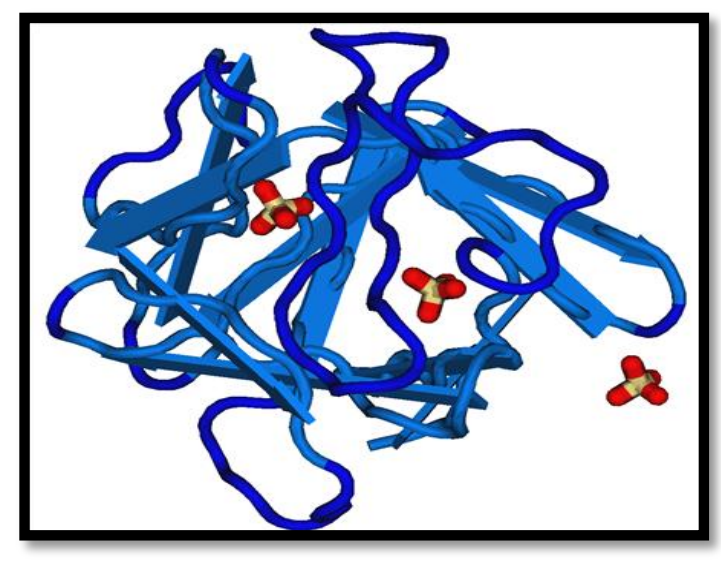

Figure 1: Chemical Structure of FGF

\section{SOURCES}

Both FGF-1 and FGF-2 were initially isolated from neural tissue but have been subsequently found in numerous other tissues. ${ }^{5}$ FGF-2 has been shown to be produced in the brain, pituitary gland, kidney, corpus luteum, and adrenal gland. ${ }^{7}$

\section{FUNCTIONS (Figure 2)}

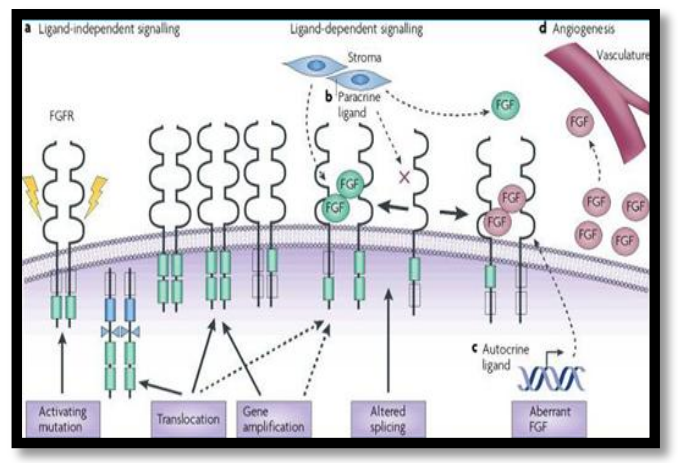

Figure 2: Functions of FGF

FGFs are multifunctional regulatory peptides with a great impact on studies of tumorigenesis, cardiovascular disease, repair of tissue injury, neurobiology and embryonic development. ${ }^{34}$ They are responsible for critical functions in wound healing, tissue repair, angiogenesis, and homeostatic regulation. ${ }^{8}$

- New blood vessel formation (Angiogenesis): FGF-2, in particular has the ability to induce all steps necessary for new blood vessel formation both in vivo and in vitro. ${ }^{6}$ FGF-1 stimulates endothelial cell proliferation. ${ }^{9}$ FGF-2 actively regulates the production of type I collagen and laminin by PDL cells. Laminin is one of the most important biological substances which participate in angiogenesis. On the other hand, FGF-2 has a most effective potential activity for angiogenesis. It has been observed in animal studies that where FGF-2 had been applied at the early phase of the wound healing process, many newly formed capillaries were observed around that area. This suggests that the production of laminin by PDL cells which is a reaction for the stimulation of FGF-2 may be helpful to angiogenesis in periodontal tissues. ${ }^{4}$

- Wound Repair: FGF stimulates the proliferation and/ or migration of most of the major cell types 
involved in wound healing, including capillary endothelial cells, vascular endothelial cells, fibroblasts, keratinocytes, epithelial cells and specialized cell types such as chondrocytes and myoblasts. FGF-2 also stimulates epithelialisation, fibronectin, proteoglycan and collagen synthesis. The administration of FGF-2 at the time of wound closure not only significantly increases the breaking strength of the wound but also improves the quality of the scar. ${ }^{6}$

- Development: The FGFs are believed to act as competence growth factors. This implies that FGF stimulates resting cells in GO to enter the cell cycle in G1. Once these resting cells enter the cell cycle, progression growth factors are needed to stimulate their transit through GI into the $\mathrm{S}$ phase or synthetic phase. Therefore, FGFs initiate a cascade of cellular events but require synergistic action with progression growth factors to maximize DNA synthesis and cell growth. ${ }^{1}$

FGFs play a role in skeletal muscle development and in lung maturation. For example, FGF-6 and its receptor induce myoblast proliferation and suppress myocyte differentiation, providing a supply of proliferating myocytes. FGF-2 is also thought to be involved in the generation of angioblasts during embryogenesis. FGF-1 and FGF-2 are involved in the specification of the liver from endodermal cells.

- Hematopoiesis: FGFs have been implicated in two aspects of hematopoesis- development of specific lineages of blood cells and the development of bone marrow stroma. ${ }^{6}$

- Cellular role: FGFs have an ability to bind to heparin and heparan sulphate. Immunohistochemical analysis of tissues for FGF-2 often reveals - FGF-2 in association with the extracellular matrix and in basement membranes is attached to heparan sulphate, ${ }^{3}$ which provides protection from degradation and allows it to maintain its biological potential. ${ }^{7}$

- Effect on bone: FGFs stimulate bone cell replication, but under some conditions can inhibit matrix synthesis by bone cells. In vivo FGF has been shown to increase bone formation, and accelerate the rate of fracture repair. ${ }^{2}$ FGF-2 stimulates periosteum derived cells in early stages of bone healing. ${ }^{8}$ Some of these effects may be mediated through increases in transforming growth factor- $\beta$ production. ${ }^{2}$ Systemic administration of FGF stimulates bonefracture healing, induces new bone formation, and increases bone mass, suggesting that there may be a therapeutic role for FGF in the treatment of osteopenia. Also, rapid bone healing has been induced in animal models by FGF- $2 .{ }^{8}$

- Role in periodontium: The stimulatory effects of FGFs on neovascularization, in addition to the chemotactic and mitogenic effects on mesodermal cells, in particular to fibroblasts and osteoblasts, suggest an important role of these proteins in periodontal wound healing and regeneration. ${ }^{1}$ Studies on the effects of FGF on individual cell types have shown that it can stimulate endothelial cell and periodontal ligament cell migration and proliferation. ${ }^{2}$

FGF-2 is widely distributed in nearly all periodontal tissues, including gingiva, periodontal ligament and bone. FGF-2 has been reported to stimulate periodontal ligament and endothelial cell migration; increases DNA synthesis and inhibits the alkaline phosphatase production of periodontal ligament cells. ${ }^{9}$ FGF-2 enhances the proliferation of the PDL cells in a dose-dependent manner and the response reaches plateau at the concentration of $10 \mathrm{ng} / \mathrm{ml}$. In addition, the proliferative response is decreased when the concentration on FGF-2 is over $100 \mathrm{ng} / \mathrm{ml}$. In contrast, alkaline phosphatase activities by PDL cells are completely inhibited at concentrations more than $10 \mathrm{ng} / \mathrm{ml}$. Though FGF-2 induces proliferation but it inhibits 
cytodifferentiation of PDL cells into osteogenic cells. It also abrogated the synthesis of type I collagen, which is one of the most common extracellular matrices in the periodontium and is essential for calcified nodule formation. ${ }^{4}$ FGF-2 may stimulate the release of proteoglycans via the increase in MMP-3 production. ${ }^{10}$ Thus they stimulate proliferation of most of the major cell types involved in wound healing both in vitro and in vivo, including vascular endothelial cells, fibroblasts, keratinocytes, chondrocytes and myoblasts. $^{3}$

In-vivo studies have demonstrated that a topical application of exogenous FGF-2 enhances the healing process of duodenal ulcers, chronic pressure sores, and bone fractures. Furthermore, recently it has been reported that local application of exogenous FGF-2 enhanced periodontal regeneration following experimental alveolar bone defects in beagle dogs and primates. In those experiments, it was noteworthy that no epithelial downgrowth, ankylosis, or root resorption was observed in the FGF-2 applied sites. This strongly suggests that FGF-2 plays an important role in the process of periodontal tissue regeneration, and it may also influence or regulate the functions of gingival epithelial cells, which are important components of periodontal tissue. $^{7}$

\section{CONCLUSION}

Fibroblast growth factors are a group of naturally occurring heparin-binding proteins that are potent mitogens and chemoattractants for various cells. These properties have led to the proposal that they play important roles in wound healing and regeneration of tissues. It is probable that, in the future, periodontal therapy will include root surface demineralization to expose the collagenous root matrix and topical application of a combination of polypeptide growth factors including FBFs in a slowrelease biodegradable device to promote the migration, proliferation and maturation of specific progenitor cell populations capable of regenerating the periodontium.

\section{REFERENCES}

1. Raul G. Caffesse and Carlos R. Quinones. Polypeptide growth factors and attachment proteins in periodontal wound healing and regeneration. Perio 2000. 1993;1:69-79.

2. David L. Cochran \& John M. Wozney. Biological mediators for periodontal regeneration. Perio 2000. 1999;19:40-58.

3. S Raja, G Byakod and P Pudakalkatti. Growth factors in periodontal regeneration. Int J Dent Hygiene 7, 2009;82-89.

4. Takayama S, Murakami S, Miki Y, Ikezawa K, Tasaka S, Terashima A, Asano T, Okada H. Effects of basic fibroblast growth factor on human periodontal ligament cells. J Periodont Res. 1997;32:667-675.

5. Laurie K. McCauley and Martha J. Somerman. Biologic modifiers in periodontal regeneration. DCNA April 1998: Vol 42; No. 2.

6. Vinay Kumar, Abul K. Abbas and Nelson Fausto. Robbins and Cortan Pathologic Basis of Disease. Pennsylvania: 2004.

7. Shin-ichi Takayama etal. Effect of basic fibroblast growth factor on human gingival epithelial cells. J Periodontol 2002;73:1467-1473.

8. Mehmet Akif Eskan and Henry Greenwell. Theoretical and Clinical Considerations for Autologous Blood Preparations: Platelet-Rich Plasma, Fibrin Sealants, and Plasma-Rich Growth Factors. Clin Adv Periodontics 2011;1:142-153.

9. Outline of Periodontics. $4^{\text {th }}$ edition. By J.D.Manson and B.M.Eley

10. Shimazu A, Morishita M. Basic fibroblast growth factor induces the expression of matrix metalloproteinase-3 in human periodontal ligament cells through the MEK2 mitogen-activated protein kinase pathway. J Periodont Res 2003; 38:122-129. 